\title{
Thrombopoiesis: At a Glance!
}

\author{
Preeti Tripathi $^{1} \cdot$ Arijit Sen $^{1} \cdot$ H. P. Pati $^{2}$
}

Received: 26 September 2019/Accepted: 31 December 2019/Published online: 8 January 2020

(c) Indian Society of Hematology and Blood Transfusion 2020

Megakaryocytes are stem cell derived progenitors which give rise to platelets through successive steps of differentiation. They undergo a unique maturation process which includes nuclear polyploidization, development of internal membrane system and formation of proplatelet processes from which new platelets are shed into vascular sinusoids to reach peripheral blood [1]. The nuclear remnant is then phagocytosed by the marrow macrophages. Figure 1 shows one such megakaryocyte found in the bone marrow aspirate of a patient with immune thrombocytopenia wherein the megakaryocytic cytoplasm is extended into long serpentinous proplatelet processes (also known as platelet ribbons). The megakaryocytic nucleus is seen at one side and multiple new platelets (fragments of cytoplasm) are observed at the ends of these ribbons. As megakaryocytes progressively differentiate, they become polyploid due to endomitosis. This polyploidization facilitates massive protein production required for the formation of large number of platelets (one megakaryocyte gives rise to up to 5000 platelets). This platelet shedding into marrow sinusoids was first demonstrated in vivo using fluorescently labelled megakaryocytes [2]. Such a phenomenon is rarely visible on aspirate smears. This

Preeti Tripathi

contactdoctorpreeti@gmail.com

Arijit Sen

aseniaf@gmail.com

H. P. Pati

harappati@yahoo.co.in

1 Department of Laboratory Medicine, Command Hospital Airforce Bengaluru, Room No 21, Second Floor, Bangalore 560008, India

2 Department of Hematology, All India Institute of Medical Sciences, New Delhi, New Delhi, India

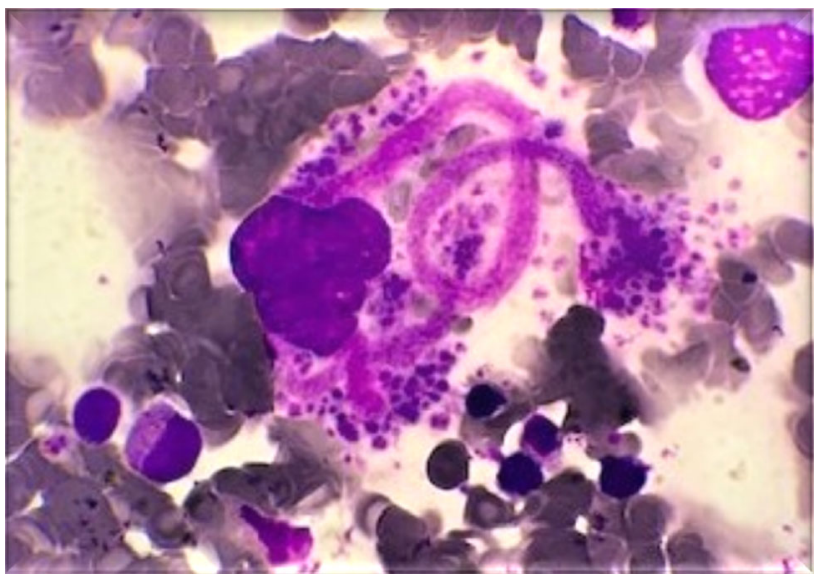

Fig. 1 Leishman-Giemsa stained Bone marrow aspirate smears $(1000 \times$ oil immersion) showing mature megakaryocyte with cytoplasmic extensions (proplatelet processes or Platelet Ribbons) releasing new platelets at ends. Polyploid nuclei is seen at one side

picture depicts a transient step of megakaryocytic differentiation with a lasting visual impression.

\section{Compliance with Ethical Standards}

Conflict of interest The authors declare that they have no conflict of interest.

\section{References}

1. Machlus KR, Italiano JE (2013) The incredible journey: from megakaryocyte development to platelet formation. J Cell Biol 201(6):785-796

2. Junt T, Schulze H, Chen Z, Massberg S, Goerge T, Krueger A et al (2007) Dynamic visualization of thrombopoiesis within bone marrow. Science 317:1767-1770

Publisher's Note Springer Nature remains neutral with regard to jurisdictional claims in published maps and institutional affiliations. 